\title{
A TRANSITION REGION EXPLOSIVE EVENT OBSERVED IN He II WITH THE MOSES SOUNDING ROCKET
}

\author{
J. Lewis Fox ${ }^{1}$, Charles C. Kankelborg ${ }^{1}$, and Roger J. Thomas ${ }^{2}$ \\ ${ }^{1}$ Montana State University EPS 264, Bozeman, MT 59717, USA; fox@ physics.montana.edu, kankel@ solar.physics.montana.edu \\ ${ }^{2}$ Goddard Space Flight Center NASA/GSFC Code 671, Greenbelt, MD 20771, USA; Roger.J.Thomas@ nasa.gov \\ Received 2009 November 13; accepted 2010 June 19; published 2010 July 27
}

\begin{abstract}
Transition region explosive events (EEs) have been observed with slit spectrographs since at least 1975, most commonly in lines of $\mathrm{C}$ IV $(1548 \AA, 1550 \AA)$ and Si IV $(1393 \AA, 1402 \AA)$. We report what we believe to be the first observation of a transition region EE in He II $304 \AA$. With the Multi-Order Solar EUV Spectrograph (MOSES) sounding rocket, a novel slitless imaging spectrograph, we are able to see the spatial structure of the event. We observe a bright core expelling two jets that are distinctly non-collinear, in directions that are not anti-parallel. The jets have sky-plane velocities of order $75 \mathrm{~km} \mathrm{~s}^{-1}$ and line-of-sight velocities of $+75 \mathrm{~km} \mathrm{~s}^{-1}$ (blue) and $-30 \mathrm{~km} \mathrm{~s}^{-1}$ (red). The core is a region of high non-thermal Doppler broadening, characteristic of EEs, with maximal broadening $380 \mathrm{~km} \mathrm{~s}^{-1}$ FWHM. It is possible to resolve the core broadening into red and blue lineof-sight components of maximum Doppler velocities $+160 \mathrm{~km} \mathrm{~s}^{-1}$ and $-220 \mathrm{~km} \mathrm{~s}^{-1}$. The event lasts more than $150 \mathrm{~s}$. Its properties correspond to the larger, long-lived, and more energetic EEs observed in other wavelengths.
\end{abstract}

Key words: instrumentation: spectrographs - space vehicles: instruments - Sun: transition region - Sun: UV radiation - techniques: imaging spectroscopy

Online-only material: animation

\section{INTRODUCTION}

Transition region explosive events (EEs) have been widely observed with spectrographs such as the High-Resolution Telescope and Spectrograph (HRTS; Brueckner \& Bartoe 1983; Cook \& Brueckner 1991), and the Solar Ultraviolet Measurement of Emitted Radiation (SUMER; Innes et al. 1997, and references therein). With HRTS, they were most typically observed in C IV $1548 \AA$, $1550 \AA$. In SUMER, Si IV $1393 \AA$, $1402 \AA$ are used. Both line doublets are formed at temperatures of $\sim 100,000 \mathrm{~K}(100,000 \mathrm{~K}$ and $63,000 \mathrm{~K}$, respectively, according to Arnaud \& Rothenflug 1985). EEs can be observed across a temperature range from $20,000 \mathrm{~K}$ in $\mathrm{C}$ II to $250,000 \mathrm{~K}$ in $\mathrm{O} \mathrm{V}$ (Moses et al. 1994). EEs are characterized by large non-thermal Doppler broadenings. Values typically given are $100 \mathrm{~km} \mathrm{~s}^{-1}$ to the red and the blue (Dere 1994). EEs divide between those which are broadened to both sides of line center and those predominantly broadened to one side of line center (Cook \& Brueckner 1991; Dere et al. 1989). Events with dominant blue wings are more common than those with dominant red wings (Cook \& Brueckner 1991; Dere et al. 1989). They are typically small, $1500 \mathrm{~km}$ in length (Dere 1994; Dere et al. 1989, 1991; Cook \& Brueckner 1991). The two wings may be displaced from each other by 1000-2000 km (Dere et al. 1989; Dere 1994), although displacements up to $6000 \mathrm{~km}$ have been seen (Innes et al. 1997). The typical lifetime of an event is 60-90 s (Moses et al. 1994; Dere 1994; Dere et al. 1991). Considerable variation from "typical" properties is seen from one EE to another (Dere et al. 1989). Events are seen with velocities up to $400 \mathrm{~km} \mathrm{~s}^{-1}$ (Moses et al. 1994; Dere et al. 1989), sizes up to 4000-5000 km (Innes et al. 1997; Cook \& Brueckner 1991), and lifetimes up to 4-5 minutes (ibid). One extremely large event has been seen with a length of $\sim 20,000 \mathrm{~km}$ (Brueckner et al. 1988).

EEs in active regions coincide with emerging flux, while EEs in the quiet Sun concentrate in or near the quiet-Sun network above regions of canceling flux (Dere et al. 1991). They are typically located along a magnetic neutral line (ibid).
These features suggest that EEs are a signature of magnetic reconnection in the transition region. It has been postulated that reconnection processes producing bipolar jets are responsible for EEs (see, for example, the illustration in Figure 2 of Dere et al. 1991). The red and blue wing velocities are identified with the Alfvén speed at the reconnection site. Innes et al. (1997) report three events in which raster scanning of the SUMER slit clearly shows the bi-directional jet structure. They found that the Doppler shifts change sign from red to blue within $2-3000 \mathrm{~km}$, the wings move away from the region where the sign change occurs, and the brightest emission is found at the sign change.

We will discuss the Multi-Order Solar EUV Spectrograph (MOSES) instrument, and the 2006 February 8 rocket flight during which a compact EE was observed in He II $304 \AA$. We will demonstrate what can be learned about the event from imaging data alone and show the magnetic context of our EE, which has two canceling bipoles under it. We use an indirect spectral analysis technique we call "parallax analysis," described in Section 3.3, to infer the Doppler shifts, and line width along the $\mathrm{N}-\mathrm{S}$ axis of the event, perpendicular to the dispersion direction of MOSES. The He II $304 \AA$ event we report here is a bidirectional jet from sky-plane motion as well as Doppler shift. We will show that its properties fit within the envelope found by previous authors and it may properly be identified as a transition region EE. We believe this to be the first He II $304 \AA$ EE reported in the literature (an EE in He II at $256 \AA$ was reported by Dere et al. 2007). However, contrary to previous models, we find that the structure is not collinear and that the jets are not anti-parallel.

\section{MOSES}

The MOSES rocket is a novel multi-order slitless imaging spectrometer. MOSES provides high spatial resolution (0''6 pixels), high cadence ( $<10 \mathrm{~s}$ ), wide field-of-view (FOV, $10^{\prime} \times$ $20^{\prime}$ ) images in a narrow EUV spectral band around $304 \AA$. Images are recorded at three spectral orders $m=+1,0,-1$, allowing spectral information to be disentangled from spatial 


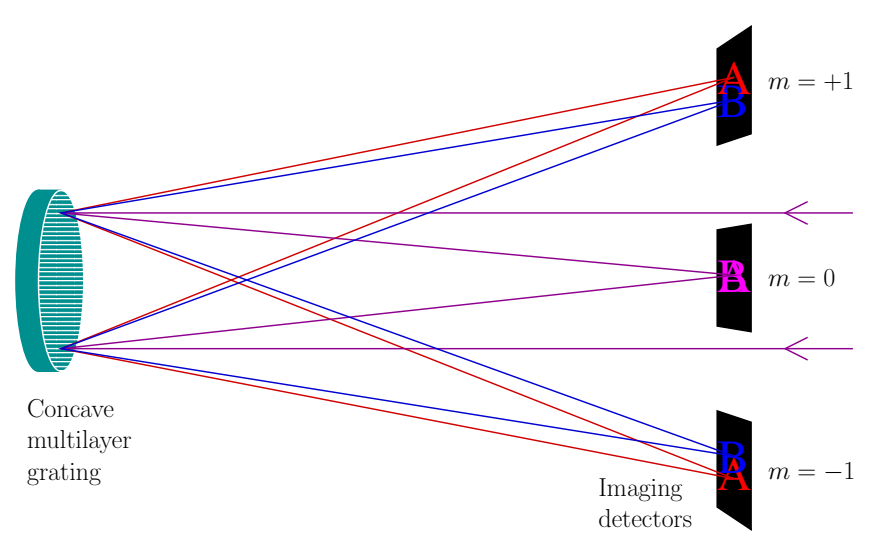

Figure 1. Conceptual sketch of MOSES.

information. Unlike slit-scanning or filter tuning techniques, data are cotemporal at every point in all wavelengths.

\subsection{Instrument Concept}

The MOSES concept, illustrated in Figure 1, was first described by Kankelborg et al. (2000) and by Kankelborg \& Thomas (2001). The instrument is a slitless, objective grating spectrograph. By taking images at three spectral orders $m=+1,0,-1$, it is possible to acquire spatial and spectral information simultaneously over a wide FOV. The passband is limited to a few spectral lines by multi-layer coatings so that the data inversion for spectral information is tractable. In principle, detectors may be placed at any number of spectral orders. If more orders are recorded, more spectral information will be obtained.

We will now show that a direct correspondence exists between tomography and MOSES. Our instrument performs tomography on a "hyper-spectral" object with two spatial dimensions $x$ and $y$ and one spectral dimension $\lambda$. The three MOSES orders correspond to three different look-angles in conventional tomography.

The diagram in Figure 1 shows only the objective grating and detectors. It displays the effect of imaging an object which, for illustrative purposes, contains only the monochromatic letters "A" (redward of passband center) and "B" (blueward of passband center). In the $m=0$ order, the letters are projected onto the same spatial location and overlap. In the $m=+1,-1$ orders,

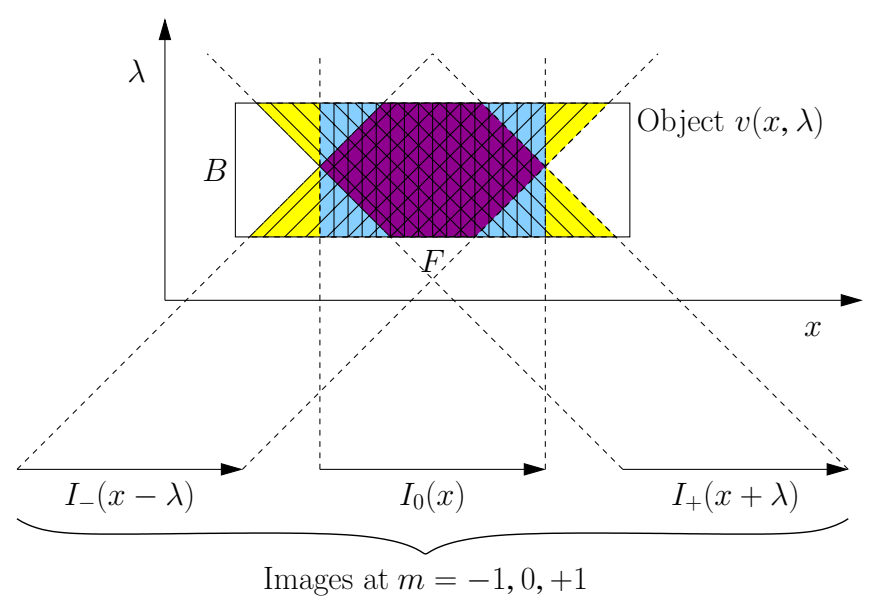

Figure 2. Diagram demonstrating how Equation (1) produces the three MOSES data orders by projections through the volume of an optically thin hyper-spectral object, mathematically equivalent to tomographic projection at three angles. The orders are indicated as well as the FOV and passband domains $F$ and $B$. The colors indicate the number of overlapping orders which sample a given region of $(x, \lambda)$ space. Similar constructions apply for each $y$-slice of the image perpendicular to the spectrograph's dispersion plane.

the spectral dispersion of the grating causes the letters to be separated on the detector by a distance proportional to the wavelength shift between them. Although they still overlap, as will be the case for any extended object, this separation allows us to infer the spectral content of the sources observed in the undispersed $(m=0)$ image. Note that the information in the $m=+1$ and $m=-1$ orders is not redundant. The direction of the $+x$ spatial axis is unchanged (the letters are not flipped) but the wavelength axes are oppositely directed, causing us to read " $A$ " in one order but $" B$ " " in the other.

We generalize this discussion with the projection equation (Equation (1)) depicted graphically in Figure 2. The equation is cast in a form that allows consideration of a system with any number of spectral orders. The treatment is readily generalizable to include dispersion in the vertical as well as horizontal directions. An object $v(x, y, \lambda)$ forms images at spectral orders $m$ :

$$
I_{m}\left(x^{\prime}, y^{\prime}\right)=\int_{B} v\left(x^{\prime}-m \lambda, y^{\prime}, \lambda\right) d \lambda
$$

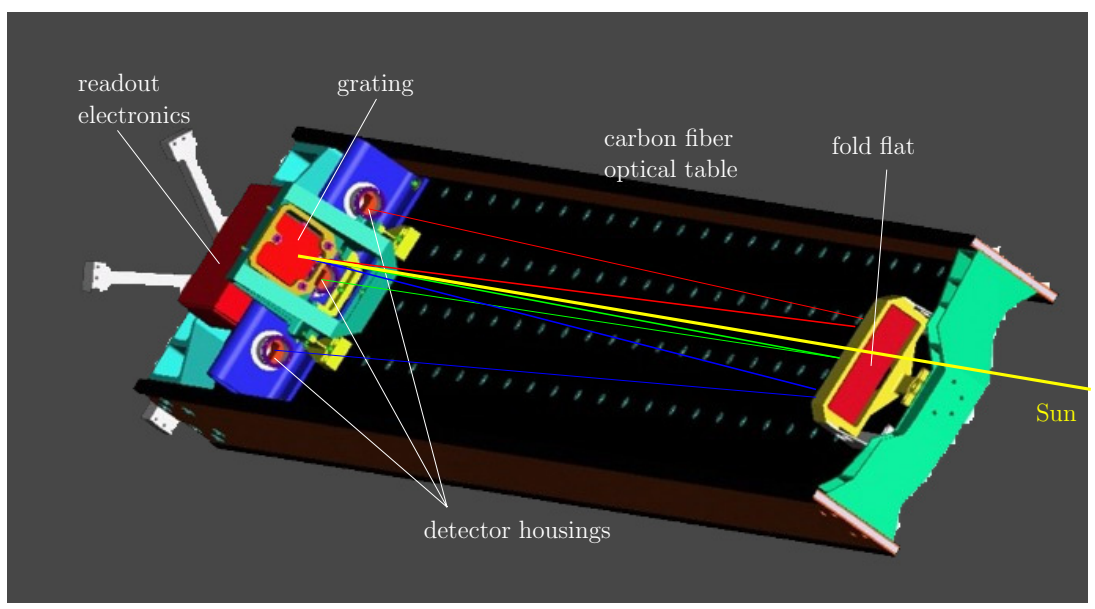

Figure 3. Layout of the MOSES instrument. Not shown are the baffles, front aperture plate, shutter, and LN2 plumbing. Most of the payload electronics are on the opposite side of the optical table. The colored lines show principal optical rays. The yellow ray comes from the Sun. The red, green, and blue rays show the path of light going to the $m=+1, m=0$, and $m=-1$ orders, respectively. 
where $\left(x^{\prime}, y^{\prime}\right)$ are the detector coordinates and domain $B$ represents the passband of the instrument. ${ }^{3}$ For the MOSES rocket experiment, $m=(+1,0,-1)$. For convenience, position $\left(x^{\prime}, y^{\prime}\right)$ on the detector, position $(x, y)$ on the Sun, and wavelength $\lambda$ are all measured in pixels. The kernel is a projection operator. For example, in the $m=+1$ order, a pixel at position $\left(x^{\prime}, y^{\prime}\right)$ receives light integrated along a slice $x+\lambda=x^{\prime}$ in the $x \lambda$-plane with $y=y^{\prime}$ a constant (compare Figure 2).

Once the spectral orders are intercalibrated, the projections of $v(x, y, \lambda)$ obey a normalization condition,

$$
N=\int_{F} I_{m} d x=\int_{B} \int_{F} v d x d \lambda,
$$

for all orders $m$. The normalization condition holds exactly if the FOV is defined by a field stop prior to the dispersive element. Otherwise, the projections of the three orders will cover slightly different domains in $(x, y, \lambda)$ at the edges of the FOV, as shown in Figure 2.

\subsection{Optics}

The optical configuration of MOSES is illustrated in Figure 3. The primary mirror is a concave spherical diffraction grating operated $1^{\circ}$ off-axis, with focal length $4.74 \mathrm{~m}$, and $8 \mathrm{~cm}$ square clear aperture $(f / 59)$ with a pitch of 950 lines $\mathrm{mm}^{-1}$, giving a reciprocal dispersion of $29 \mathrm{~m} \AA\left(29.6 \mathrm{~km} \mathrm{~s}^{-1}\right)$ per pixel at $304 \AA$. The pixel subtent in this optical system is 0.6 or $440 \mathrm{~km} \mathrm{pixel}^{-1}$ on the Sun. The design was ray traced and optimized by Roger J. Thomas at Goddard Space Flight Center (GSFC; Thomas \& Kankelborg 2001). The major optical elements are shown in Figure 3. Thin film aluminum filters (not shown) cover the open end of each detector housing to make a light tight box.

The grating flown on MOSES is a Zerodur substrate with laminar rulings fabricated by Carl Zeiss Laser Optics (Owens et al. 2005). Precise control of the groove depth allows an optimal distribution of light in the three central spectral orders, where our detectors are placed. The fold flat is Ultra-Low Expansion (ULE) glass with a protected aluminum coating on the reverse side for instrument alignment. Multi-layer $\mathrm{B}_{4} \mathrm{C} /$ $\mathrm{Mg}_{2} \mathrm{Si}$ coatings were applied to the gratings and fold flat at GSFC. The coatings provide a high reflectivity $(\approx 0.4)$, narrow passband peaked at $304 \AA$. The band contains two strong solar emission lines: He II $303.8 \AA$ and Si XI $303.3 \AA$ A. Nearby contaminant lines at $284 \AA$ and $335 \AA$ are suppressed by factors of 92.7 and 223.4 in $m=+1,98.6$ and 51.0 in $m=0$, and 76.4 and 137.7 in $m=-1$, respectively (see Owens et al. 2005, Tables 2(a) and (b)).

The optics are mounted on the Lockheed Optical Table System (LOTS) developed by Marilyn Bruner for the Solar Plasma Diagnostics Spectrometer mission (Bruner et al. 1989). This system provides a very stable, strong, lightweight, low thermal-expansion structure capable of space flight but with all the advantages of an optical test bench. The layout of the optics and detectors on LOTS is shown in Figure 3.

\subsection{Detectors}

The detectors are three $2048 \times 1024$, rear-illuminated E2V CCD42-20 devices at spectral orders $m=+1,0,-1$. The readout electronics are a three-channel variant of the Hinode/EIS system, contributed by Mullard Space Science Laboratory (MSSL). The power supply is also a copy of the corresponding EIS system (Kosugi et al. 2007; Culhane et al. 2007).

\footnotetext{
3 We find this formulation more natural than the notation developed previously in Kankelborg \& Thomas (2001).
}

Thermoelectric coolers regulate CCD temperature to $-30^{\circ} \mathrm{C}$. An aluminum block, kept cold with liquid nitrogen (LN2) until launch, is the heat dump for the thermal control system.

\subsection{Electronics}

Data handling and experiment control are through a single experiment computer, a commercial off-the-shelf (COTS) EBX CPU board running a minimal Linux distribution. A PC/104+ stack on the CPU board contains COTS cards for serial communications, camera data interface, and high-speed telemetry. The camera data interface is a flexible I/O (FIO) board interfacing an FPGA to the computer PC/104+ buss. We programmed the FPGA to interface to MSSL supplied electronics. All science data are stored onboard in a $1 \mathrm{~GB}$ flash disk. As much science data as possible are sent to high-speed telemetry during flight. Serial uplink and downlink channels allow two-way communication with the experiment computer.

\section{DATA AND ANALYSIS}

MOSES launched from White Sands Missile Range, New Mexico, 2006 February 8 at 18:44 UT. Approximately 5 minutes of observing time above $160 \mathrm{~km}$ resulted in 27 exposures taken in the interval 18:45:54-18:50:50 UT. The $10^{\prime} \times 20^{\prime}$ FOV extends from above the east limb to just past disk center. In this paper, we analyze only data from the $m=0$ and $m=+1$ spectral orders. Data have been dark-subtracted. We have co-aligned the two orders and removed optical distortion (see the Appendix).

Intensities are given in units of data numbers (DN) per second, normalized so that different frames have the same mean $\mathrm{DN} \mathrm{s}^{-1}$ as the exposure closest to apogee. The following equations describe the normalization process:

$$
\begin{gathered}
I_{0}^{\prime j}=\frac{\left\langle I_{0}^{a}\right\rangle}{\left\langle I_{0}^{j}\right\rangle} I_{0}^{j}, \\
I_{ \pm 1}^{\prime j}=\frac{\left\langle I_{0}^{a}\right\rangle}{\left\langle I_{ \pm 1}^{a}\right\rangle} \frac{\left\langle I_{0}^{a}\right\rangle}{\left\langle I_{0}^{j}\right\rangle} I_{ \pm 1}^{j},
\end{gathered}
$$

with superscript $j$ indexing exposure number, 0-26, and superscript $a$ indicating the exposure closest to apogee, frame 13. This procedure corrects for the different throughput in each channel and the variance in effective exposure time due to atmospheric absorption over the flight. The first factor in Equation (4) is the non-time-varying relative response of the orders. The second factor (which also appears in Equation (3)) removes the effect of time-varying atmospheric absorption without model-dependent assumptions.

\subsection{Zero-order Data and Magnetic Context}

The $m=0$ channel is equivalent to a narrowband $304 \AA$ imager. On the solar disk, the passband is dominated by the He II line, which we expect to account for more than $95 \%$ of the detected photons (Thomas \& Neupert 1994; Brosius et al. 1998; Vernazza \& Reeves 1978). Figure 4 shows an $m=0$ image from frame 23 , a $6 \mathrm{~s}$ exposure taken at $\sim 18: 50: 22 \mathrm{UT}$. The area in the box is displayed in Figure 5. The EE, at $\sim 100^{\prime \prime}$ $\mathrm{E}, 180^{\prime \prime} \mathrm{N}$ of disk center, consists of a bright core and two jets. A movie of MOSES frames (available in the online journal as an extension of Figure 5) shows brightening of the core followed by the two jets shooting out from the opposite corners of the 


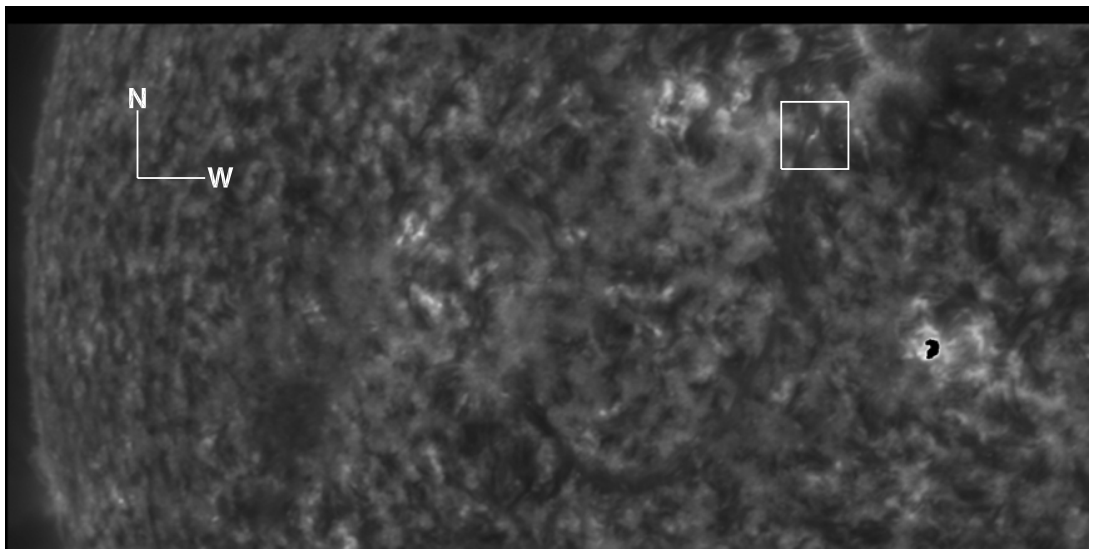

Figure 4. $6 \mathrm{~s}$ MOSES $m=0$ order image, taken 18:50:22 UT. The indicated area is shown expanded in Figure 5. A bright feature near the SW corner of the image is saturated.

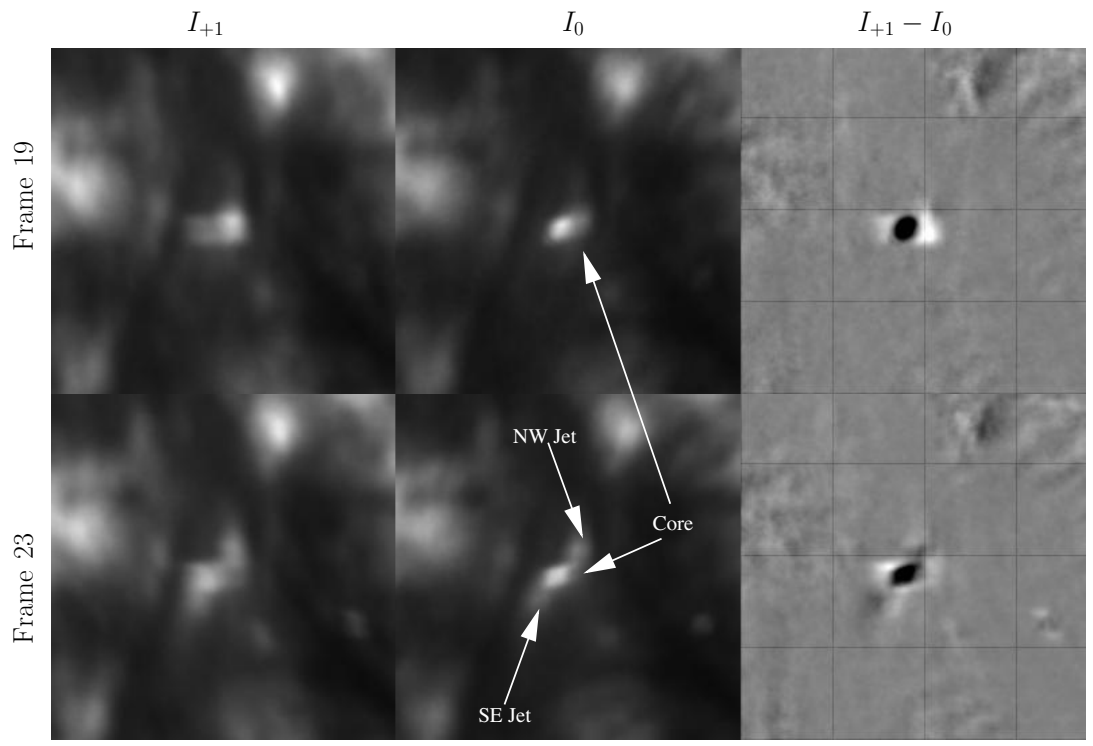

Figure 5. $m=+1$ data at left, $m=0$ at center, difference image $I_{+1}-I_{0}$ at right. The grid lines in the third (difference) panel are $20^{\prime \prime}$ apart. In the difference image, white is positive, black is negative, and gray is near zero. The top frame is from $\sim 18: 49: 15$ UT ( $3 \mathrm{~s}$ exposure) before the jets begin to emerge from the core. The bottom frame is from 18:50:22 UT (6 s exposure). At this point, the jets are well defined. This is the exposure analyzed in the rest of the paper. These two image triples are from a movie of such triples, available in the online edition, covering the entire flight data sequence from 18:45:54 UT to 18:50:50 UT. Orange pixels in the movie represent bad data.

(An animation of this figure is available in the online journal.)

core. The event is visible for $\sim 150 \mathrm{~s}$ and was still in progress when the MOSES exposure sequence ended.

Figure 6 shows light curves calculated from raw data for each of the three regions, core, NW jet, and SE jet, along with the total light curve for all three regions. The light curves are the total intensity within the appropriate box shown in Figure 7 for all 27 science exposures. Time is indexed from the beginning of the first exposure. The light curves show the core energizing before the jets. The event is not "winding down" at the time MOSES ceased data-taking. Indeed, it is not possible to say whether we observed even the middle of it. The uptick in total intensity in the last frame could indicate the beginning of a new phase in the event. The event time of $150 \mathrm{~s}$ must be taken as a lower bound.

Figure 8 shows a larger context around the event, with magnetic poles derived from MDI data and the position of a filament near the edge of the event. The "axis" of the event, as defined by the direction of the jets, appears roughly parallel to the filament. The sky-plane velocities shown in Figure 8 were calculated from centered differencing of the position of the center of each jet in frames 22 and 24 as shown in Figure 9. The resulting sky-plane velocities are $v_{\mathrm{NW}}=(12,80) \mathrm{km} \mathrm{s}^{-1}$ and $v_{\mathrm{SE}}=(17,-70) \mathrm{km} \mathrm{s}^{-1}$. The $+x$ axis is aligned with solar $\mathrm{W}$ and the $+y$ axis with solar $\mathrm{N}$. Also displayed in Figure 8 is the MDI magnetogram closest in time to our launch, $\approx 8$ minutes before the event starts. The magnetic poles labeled N47 and P18 underlie the EE. MDI magnetogram movies from the day of launch show that the poles are converging and canceling.

\subsection{Difference Images}

The spectrally interesting features of the EE can be visualized quickly with a simple difference image. Figure 5 shows in the three lower panels the $m=+1$ order data, the $m=0$ order data, and the difference $I_{+1}-I_{0}$ for the region immediately around the EE at $\sim 18: 50: 22$ UT. From the difference image, it is clear that the central bright feature of the EE is broadened, the SE jet is blue shifted, and the NW jet is slightly red shifted. The top set of panels in Figure 5 shows the same field for an earlier time. No 


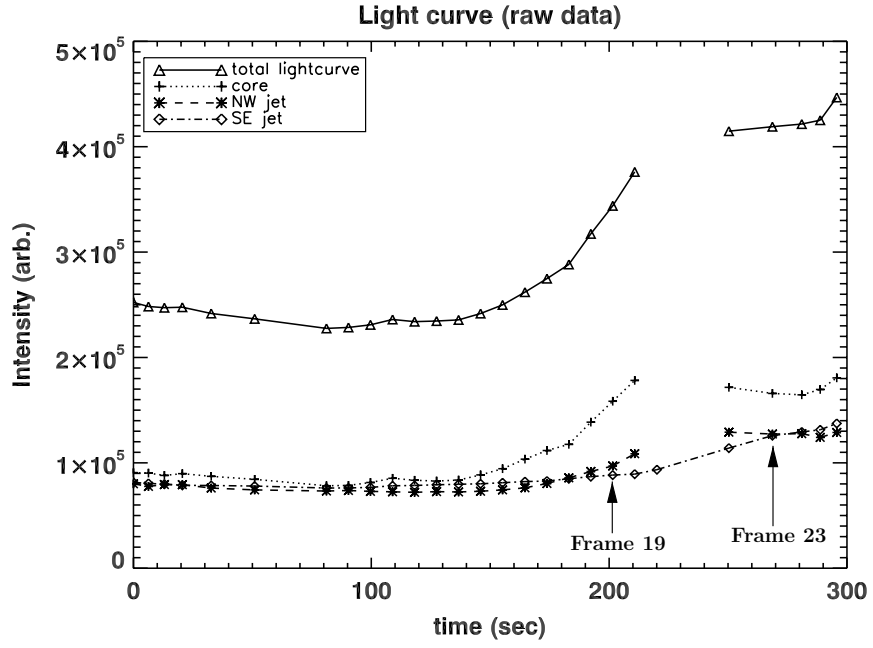

Figure 6. Light curve of the EE for the whole MOSES-06 flight. Time is measured in seconds from the beginning of the first science exposure. The intensities for each exposure are assigned to the time at which that exposure began. The curves are calculated from data without background subtraction. The regions corresponding to NW jet, core, and SE jet are shown in Figure 7. The missing point in the "total," "core," and "NW jet" curves (a 24 s exposure) is due to saturated pixels in the core and NW jet regions. Saturated pixels are marked as bad data and the sum is ignored when such pixels fall inside the regions-of-interest.

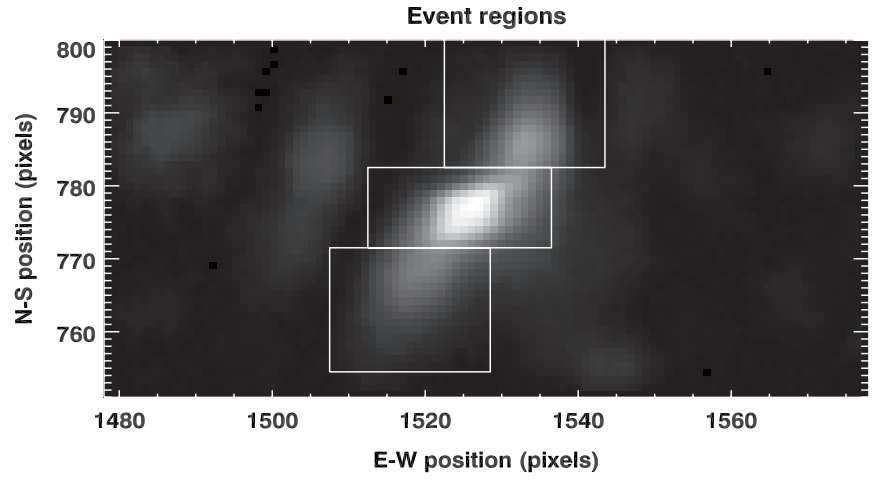

Figure 7. Regions-of-interest over which intensity totals were calculated for Figure 6 . The regions are given by the boxes. The gray-scale intensity is the $m=0$ order image from $\sim 18: 50: 22$ UT with background subtracted to increase the contrast around the event for visual clarity. The light curves were calculated from unbackgrounded data. The three regions are contiguous.

jets are yet visible, although the core brightening and broadening is clear. These two sets of panels are extracted from a movie, showing the time evolution of the EE, which is available in the online edition of this journal. It is also clear from the difference image that the event is visible in the He II line and is not a $\mathrm{Si}$ XI feature. The difference between He II and Si XI line centers amounts to a shift of $\sim 17$ MOSES pixels between $m=+1$ and $m=0$ orders. Such a large shift would be unmistakable in the difference image.

\subsection{Parallax Analysis}

Because MOSES has no slit, spectral line information is inferred from differences between orders rather than observed directly. We expect full inversions of the MOSES data to provide line profile information for every pixel. Preliminary work along these lines has been encouraging and is reported elsewhere (Fox \& Kankelborg 2002; Fox et al. 2003; Kankelborg \& Fox 2004). In this paper, we perform a more direct analysis, which we
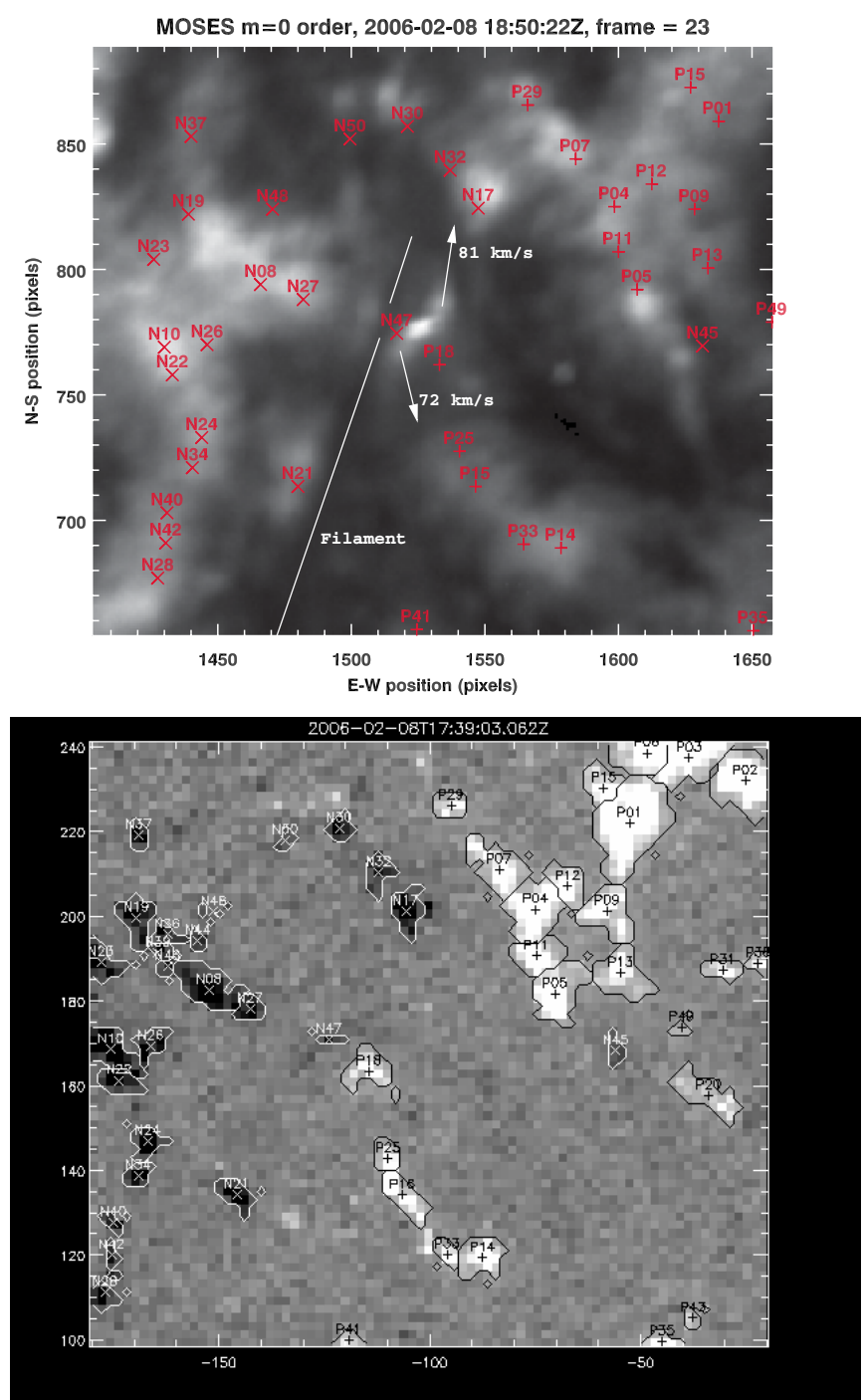

Figure 8. MOSES order $m=0$ on top, MDI magnetogram on bottom. Downhill gradients have been used to label flux concentrations. The magnetic centroids indicated in the MDI figure are shown on the $m=0$ image with their positions adjusted for the time difference between the images. The axes on the MDI figure are $\operatorname{arcsec} \mathrm{W}$ and $\mathrm{N}$ of disk center. The image coordinates in this and following figures displaying MOSES data are pixel units (0!'6) counted from the SE corner of the $2048 \times 1024$ detector. Arrows on the MOSES image indicate the sky-plane flow directions of the two emerging jets. Note that they are roughly parallel to the nearby filament axis, but clearly are neither collinear nor even anti-parallel to one another.

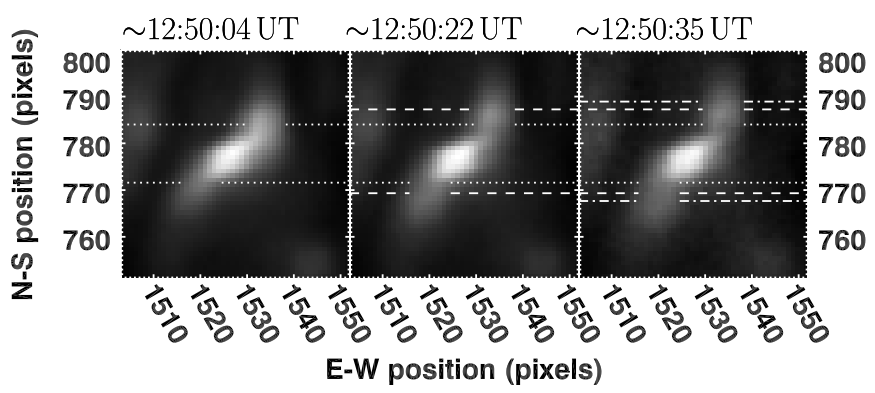

Figure 9. Three sequential $m=0$ images. 18:50:04 UT is a $12 \mathrm{~s}$ exposure; :22 $6 \mathrm{~s} ;: 35,1.5 \mathrm{~s}$. The image sequence shows the sky-plane development of the two jets. These images were used to compute the sky-plane velocities quoted in Figure 8, from the centered difference of the jet positions shown by the dotted and dash-dotted lines. The dashed line shows the jet position in the central image analyzed in this paper. 


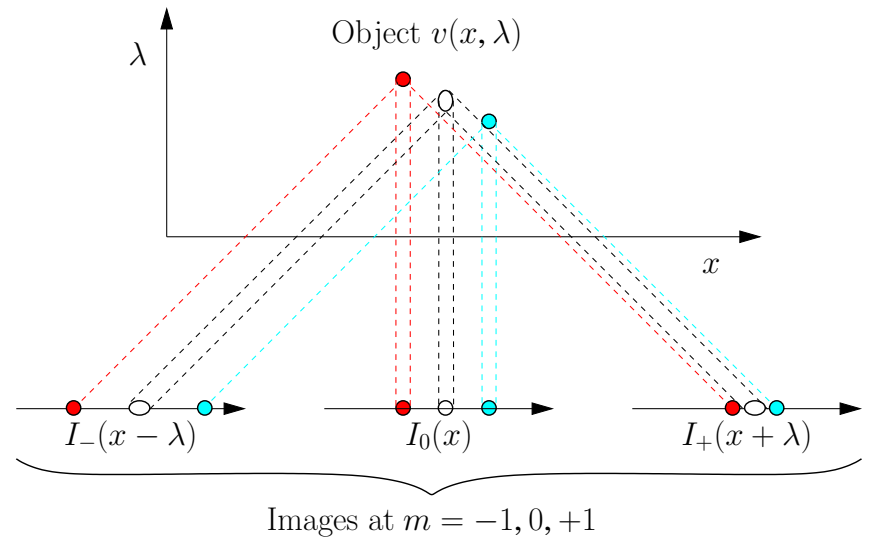

Figure 10. Revisitation of the diagram from Figure 2. Several bright compact sources are shown, demonstrating the appearance in both the hyper-spectral cube and the various data orders of a redshift, blueshift, and a broadening. For co-aligned data, the expected location in $m= \pm 1$ of a source at the central wavelength would be the same as the position of the bright source in the $m=0$ image. The parallax between $m=0$ and $m= \pm 1$ orders gives the magnitude and direction of the shift.

call "parallax analysis," that generates results independent of inversions. This type of analysis provides a check on future inversions and also generates a target list for such inversions.

As demonstrated schematically in Figure 10, a compact bright source which is shifted in wavelength (blue and red dots) jumps in position relative to a background at fixed wavelength when viewed in $m=+1$ or $m=-1$ orders. This is directly analogous to parallax. Compact sources which exhibit parallax when comparing two spectral orders (viewing angles) imply a Doppler shift. The displacement of a bright source from one order to another can be measured to infer the Doppler shift. In a similar fashion, spectral line broadening (oblong white dot in Figure 10) results in an elongation in the dispersion direction in any non-zero-order image.

\subsection{Procedure}

During its 2006 flight, the MOSES dispersion direction was closely aligned to solar E-W. We refer to this as the " $x$-axis." Solar N-S is called the " $y$-axis." Figures $11-16$ illustrate our procedure for computing the inferred Doppler shifts, which is as follows.

1. Subtract a "soap-bubble" background from the data, socalled because it mimics the behavior of a soap-bubble: it solves Laplace's equation within some boundary which is held fixed. This background is derived from a modified point-relaxation method, first described and used in Kankelborg et al. (1996) and Kankelborg et al. (1997). The data are pre-filtered using a sigma filter. The boundary pixels are held fixed and at each relaxation step any pixel values, which increased due to relaxation, are reset to their original (sigma-filtered) values (note Kankelborg et al. 1997, Appendix D states that interior pixels are only allowed to relax upward; this statement is in error). This has the effect of fixing interior local minima as well as the boundary, as though the data have "weight" and are resting on the surface of the bubble, holding it down where they touch. We use this method on the $m=0$ order data and subtract the resulting background from both $m=0,+1$. The backgrounds for three sample $y$ values are shown in Figures 11, 13, and 15, along with the raw $m=0,+1$ data.
From these examples, it is clear that the $m=0$ background is sufficient for both orders.

2. Fit slices of constant $y$ with up to 4 Gaussians. One Gaussian may not provide a sufficiently close fit to the EE data in either order. Figures 12, 14, and 16 show examples of background-subtracted data and Gaussian fits for the same three $y$-axis values. We used the Levenberg-Marquardt method of least-squares parameter estimation as implemented by William Thompson in the routine lstsqr.pro from SolarSoft (Freeland \& Handy 1998). A detailed description of the Levenberg-Marquardt method may be found in Press et al. (1992).

3. Interpret changes in a feature's position between $m=+1$ and $m=0$ images as a Doppler shift of magnitude equal to the position difference multiplied by the MOSES reciprocal dispersion of $29 \mathrm{~km} \mathrm{~s}^{-1}$ pixel $^{-1}$. Similarly, differences in feature width between images imply Doppler broadening.

4. Generate composite positions (centroids) and composite widths for purposes of comparison between orders using the formulas:

$$
\begin{gathered}
\mu_{T}=\frac{\sum_{i} \mu_{i} I_{i}}{\sum_{i} I_{i}}, \\
\sigma_{T}^{2}=\frac{\sum_{i} I_{i}\left[\left(\mu_{i}-\mu_{T}\right)^{2}+\sigma_{i}^{2}\right]}{\sum_{i} I_{i}} .
\end{gathered}
$$

The integrated intensity of the $i$ th Gaussian is denoted by $I_{i}$, the mean by $\mu_{i}$, and the variance by $\sigma_{i}^{2}$.

The interpretation of the data is often complicated. Single Gaussian peaks in $m=0$ order can split into two resolved peaks in $m=+1$ order, one to red and the other to blue. A good fit to the $m=0$ order data for the EE may require two or more Gaussians. These Gaussians may change in completely separate ways in $m=+1$ order. A fit to the EE may blur at the boundary with a weak, but wide Gaussian fit to some intensity that is not part of the event. Our best judgment has been used in determining which Gaussians belong to the event and how to treat the various parallax phenomena we see in the data. Our decisions are based on the overall appearance of the event, the spatial smoothness of the fits along the $y$-axis (across slices), the confidence level of the fits, and the temporal variation of the data. We calculate, for each order, the squared-sum of the residuals $R^{2}=\Sigma\left(I_{\mathrm{fit}}-I\right)^{2}$ and compare it to the total variance of the data $V=\Sigma I^{2}$. Here, $I$ denotes the background-subtracted data and $I_{\text {fit }}$ is the total fit (the "model," including all fitted Gaussians). The sums are performed over all image pixels in a given row. We do not use any fits that have $R^{2} / V>0.05$. Nearly all fits are well below this threshold. None of the accepted fits were close to the 5\% level. The fits which do not meet this threshold are located on the north and south edges of the event region. Visual inspection clearly establishes that they are bad fits, and the data in those areas are not well described by Gaussian functions. Therefore, the analysis is not sensitive to the choice of $5 \%$ as a maximum residual level. Dividing $R^{2}$ by the number of degrees of freedom, $N_{\text {pixels }}-N_{\text {parameters }}$, gives an estimate of the error $\sigma_{I}^{2}$ in the pixel intensity (note that this presumes a good fit, with $\chi^{2}=1$ ). This value of $\sigma_{I}$ (a constant for each slice) should be an overestimate. Feeding it to the Levenberg-Marquardt routine allows the calculation of the errors in the fit parameters, which should likewise be overestimates (see the discussion in Press et al. 1992). The "confidence level of the fit," as we have used that term here, means the confidence with which 


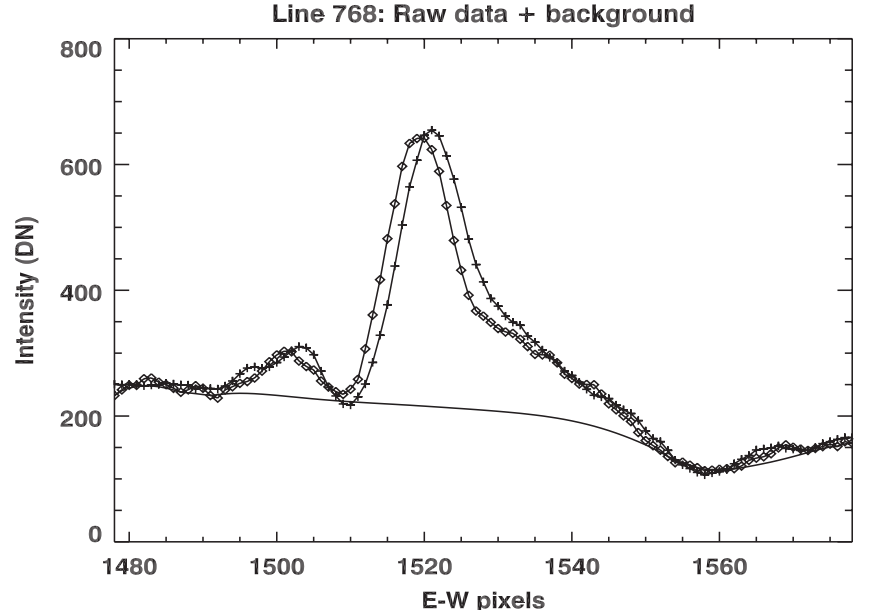

Figure 11. Raw data for $m=0$ (diamonds) and $m=+1$ orders (pluses), line 768 , with background shown by the unbroken line. These data are from the SE jet.

we exclude the zero intensity null hypothesis. Each Gaussian fit produces, among other parameters, the total integrated intensity in the Gaussian, $G_{\text {total }} \pm \sigma$. When $G_{\text {total }}<3 \sigma$, we cannot conclude with high confidence that adding that Gaussian to the fit is better than leaving it out (i.e., the total integrated intensity in that Gaussian is not significantly different from zero). In such cases, we revert to a fit with a smaller number of Gaussians. For a few fits, we have accepted confidence levels slightly less than $3 \sigma$ when doing so seemed justified by the spatial relationship to more confidently fitted Gaussians in adjacent $y$-slices.

\section{RESULTS}

The EE analyzed here exhibits three distinct regions: a bright core with non-thermal broadening (characterized by separate blueshifted and redshifted components), a blueshifted jet to the SE of the core, and a redshifted jet to the NW. Representative slices perpendicular to the $y$-axis have been taken from each of these three regions and are displayed in Figures 11-16. Each slice shown in Figures 11, 13, and 15 gives the sense of the Doppler behavior of each corresponding region by directly comparing raw $m=+1$ and $m=0$ data. The background found for the $m=0$ order from the modified point-relaxation method described in Section 3.4 is also shown. Associated with each raw data plot are plots (Figures 12, 14, and 16) showing the background-subtracted data and the Gaussian fits. Full details of all fit parameters are listed in Table 1. The statistical confidence in excluding the zero intensity null hypothesis for the fits is also given in Table 1. The confidence in excluding the zero Doppler shift null hypothesis is not given explicitly but can be estimated from the fit positions and associated errors.

Figure 17 shows a $60^{\prime \prime} \times 30^{\prime \prime}$ region around the event from the same frame as Figure 8 with $m=0$ order displayed in gray scale and Gaussian fit parameters overlayed in color. Fits were only made to data that fell between the solid (dotted) white lines for $m=0(m=+1)$. These lines are called "fit limits" in the figure legend. The symbols mark the centroid position of each fit. Offsets between + and $\diamond$ symbols are Doppler shifts, with a 1 pixel $=29 \mathrm{~km} \mathrm{~s}^{-1}$ scale. The colored lines show the FWHM of each Gaussian. The color of the symbols and lines gives the peak Gaussian intensity. Widths and shifts are largely consistent from each $y$-slice to the next. It is possible from this analysis to form a coherent picture of the event in position and velocity space.
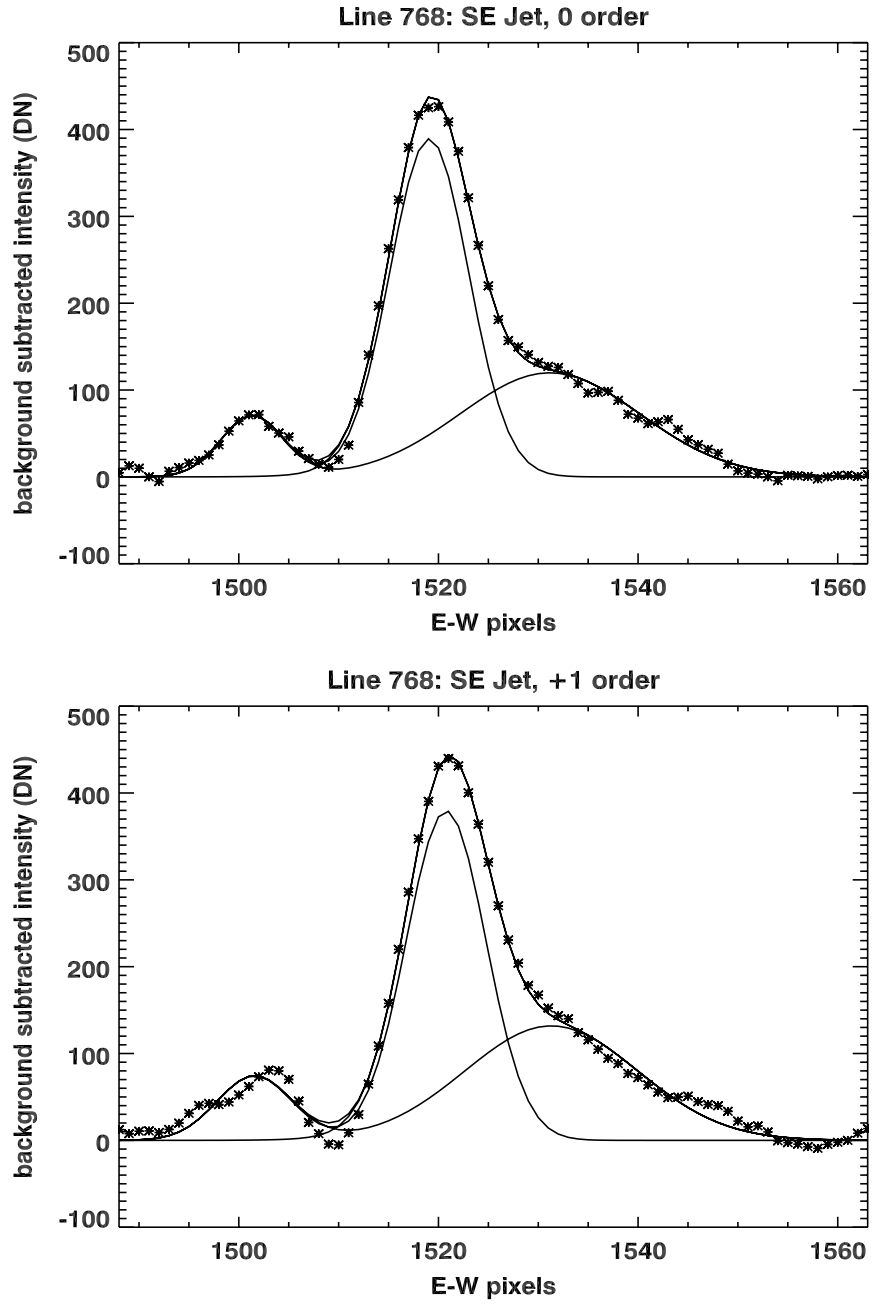

Figure 12. Background-subtracted data for line 768 with Gaussian fits overlaid for $m=0$ order (top) and $m=+1$ order (bottom). The strongest Gaussian peaks indicate a blueshift.

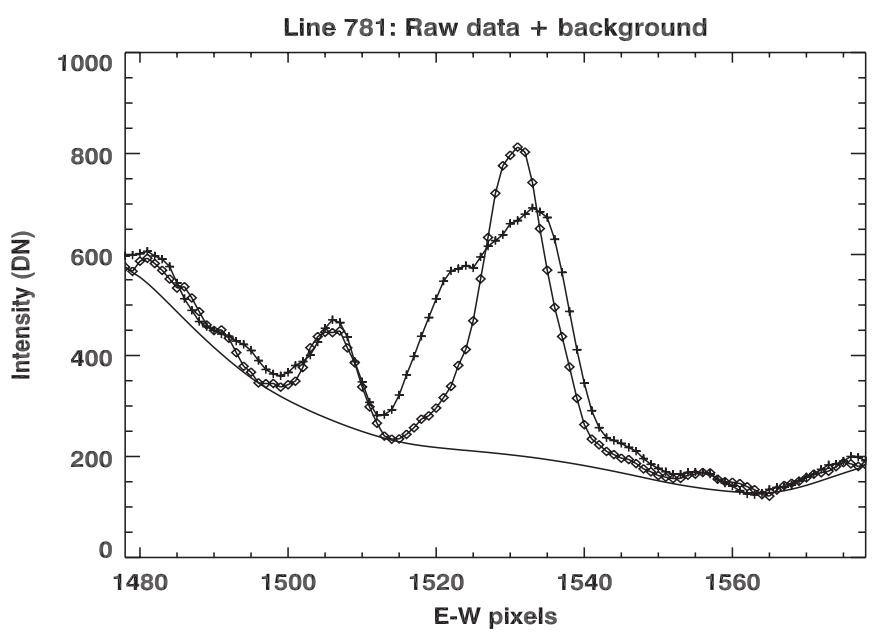

Figure 13. Raw data for $m=0$ (diamonds) and $m=+1$ orders (pluses), line 781 , with background shown by the unbroken line. These data are from the event core.

Figure 18 graphs the inferred Doppler shift derived from Gaussian fits whose centroids fell between the dashed bars in Figure 17 (called "centroid limits" in the legend for Figure 17). The dashed bars were chosen so as to exclude from consideration fits to intensity that are clearly not part of the event, 

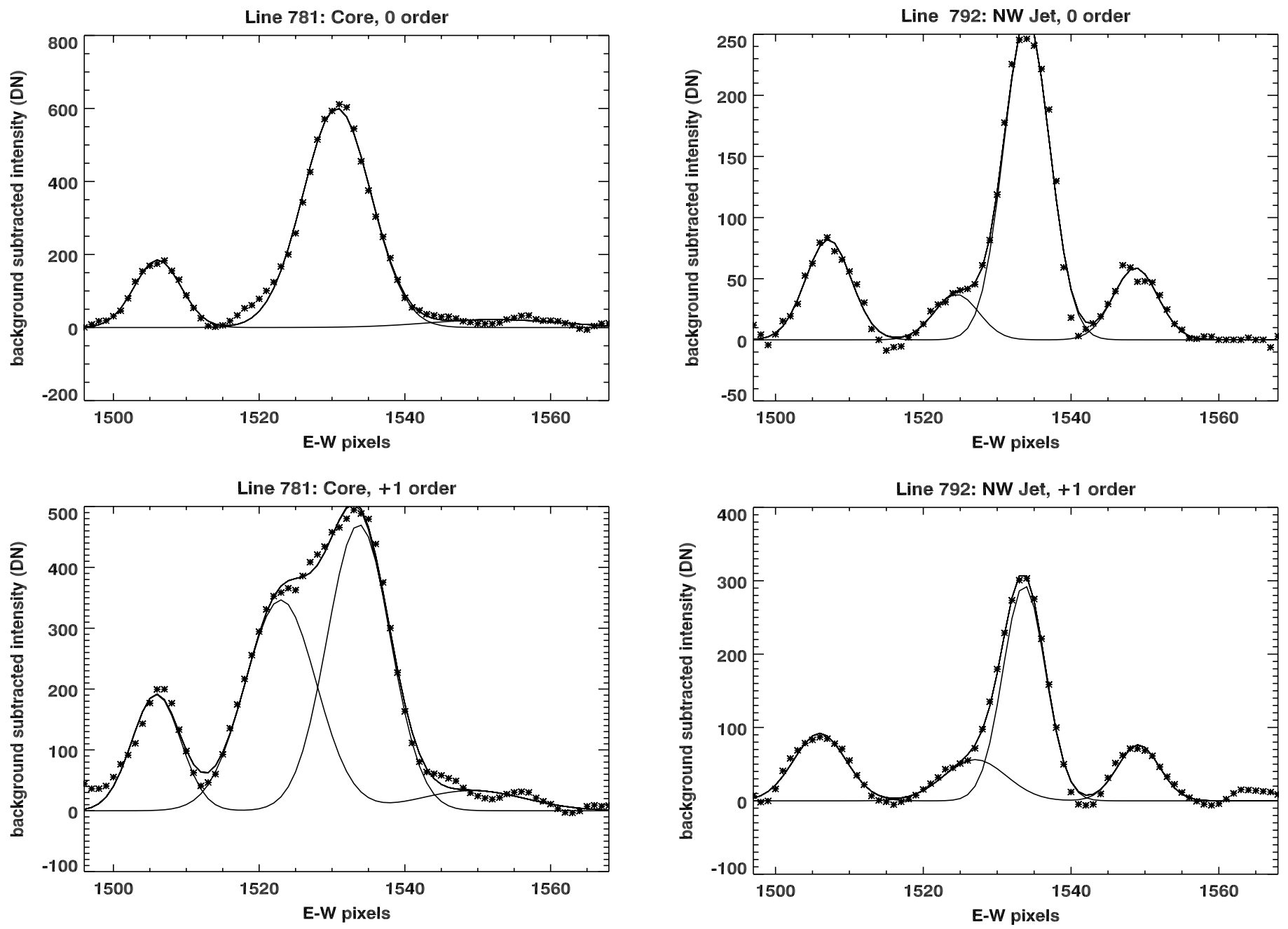

Figure 14. Background-subtracted data for line 781 with Gaussian fits overlaid for $m=0$ order (top) and $m=+1$ order (bottom). The strongest Gaussian peak in $m=0$ is in the core. In the $m=+1$ order, this single peak appears to split into clearly resolved double peaks, one to the red, and the other to the blue. We take this splitting to indicate that the non-thermal broadening in the core of this event is due to a two-component Doppler shift with both a blue and a red component.

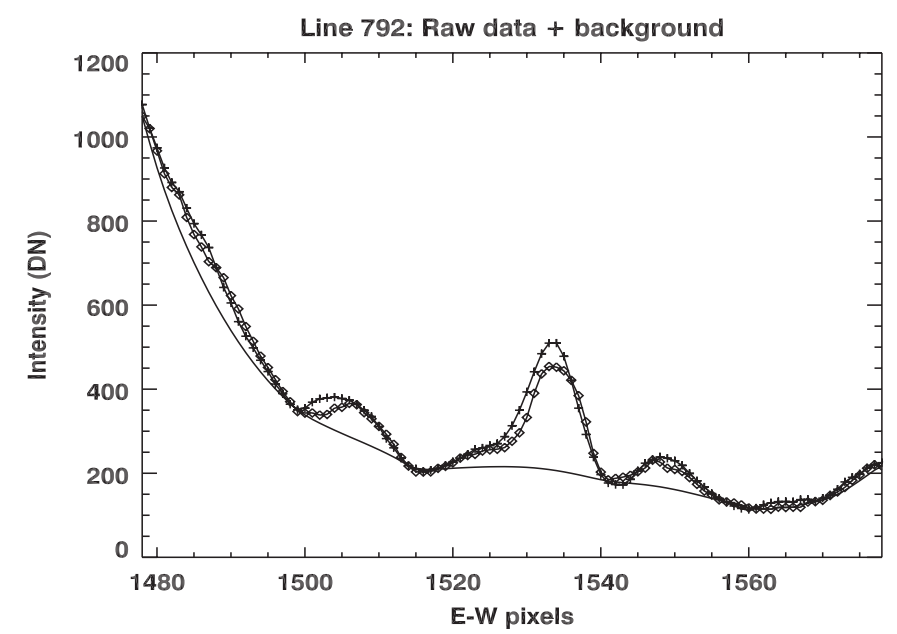

Figure 15. Raw data for $m=0$ (diamonds) and $m=+1$ orders (pluses), line 792 , with background shown by the unbroken line. These data are from the NW jet.

Figure 16. Background-subtracted data for line 792 with Gaussian fits overlaid for $m=0$ order (top) and $m=+1$ order (bottom). The strongest Gaussian peaks are located in the NW jet and show a slight, but significant redshift.

such as the linear object extending from $1490<x<1510$ and $760<y<795$ on the other side of the filament channel from the EE, or the weak intensity from 1540 to 1560 , while still including fits that might reasonably be associated with the event. The solid line in Figure 18 shows the composite Doppler shifts in the event core from $y=775$ to $y=781$ using intensity-weighted centroids found by means of Equation (5). The figure also shows Doppler velocities calculated by interpreting the Gaussian splitting as separate blueward and redward velocity components. Two fit behaviors are seen in this region. In $[1,2]$ component fits, the $m=0$ intensity is well fit by a single Gaussian, while the $m=+1$ intensity has two resolved Gaussians, one to each side of the $m=0$ fit. See Figure 14 for an example of this behavior. The two Doppler velocities are calculated by subtracting the $m=0$ centroid from each $m=+1$ centroid. [2,2] component fits are characterized by $m=0$ intensity requiring two Gaussians for a good fit. In $m=+1$, these two Gaussians are seen to spread apart from one another relative to their $m=0$ positions. These Doppler velocities are calculated by subtracting the nearest $m=0$ centroid from each $m=+1$ centroid. These fit behaviors show the non-thermal nature of the line broadening in the core. Thermal broadening could not cause a single Gaussian to split into two in the $m=+1$ order. The $m=+1$ fit would contain a single Gaussian with a 
background subtracted intensity (arb. units)

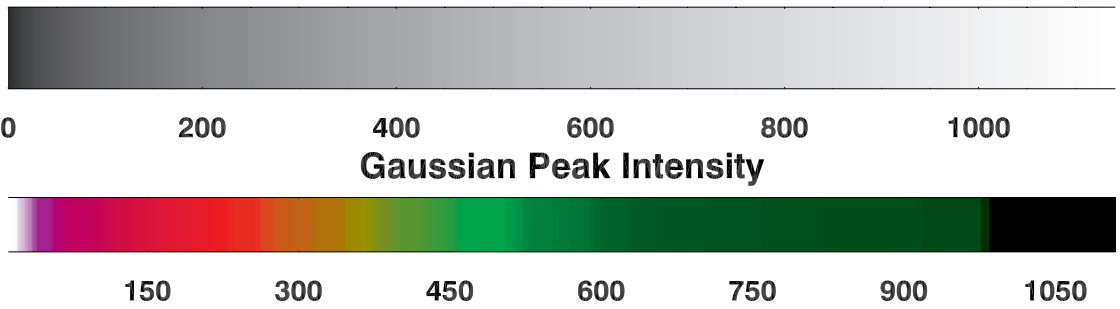

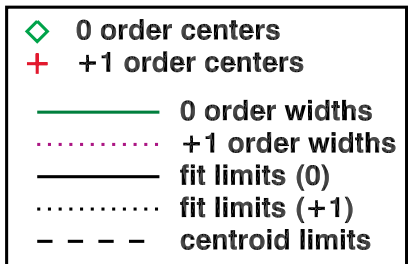

0 order (background subtracted), frame $=23$

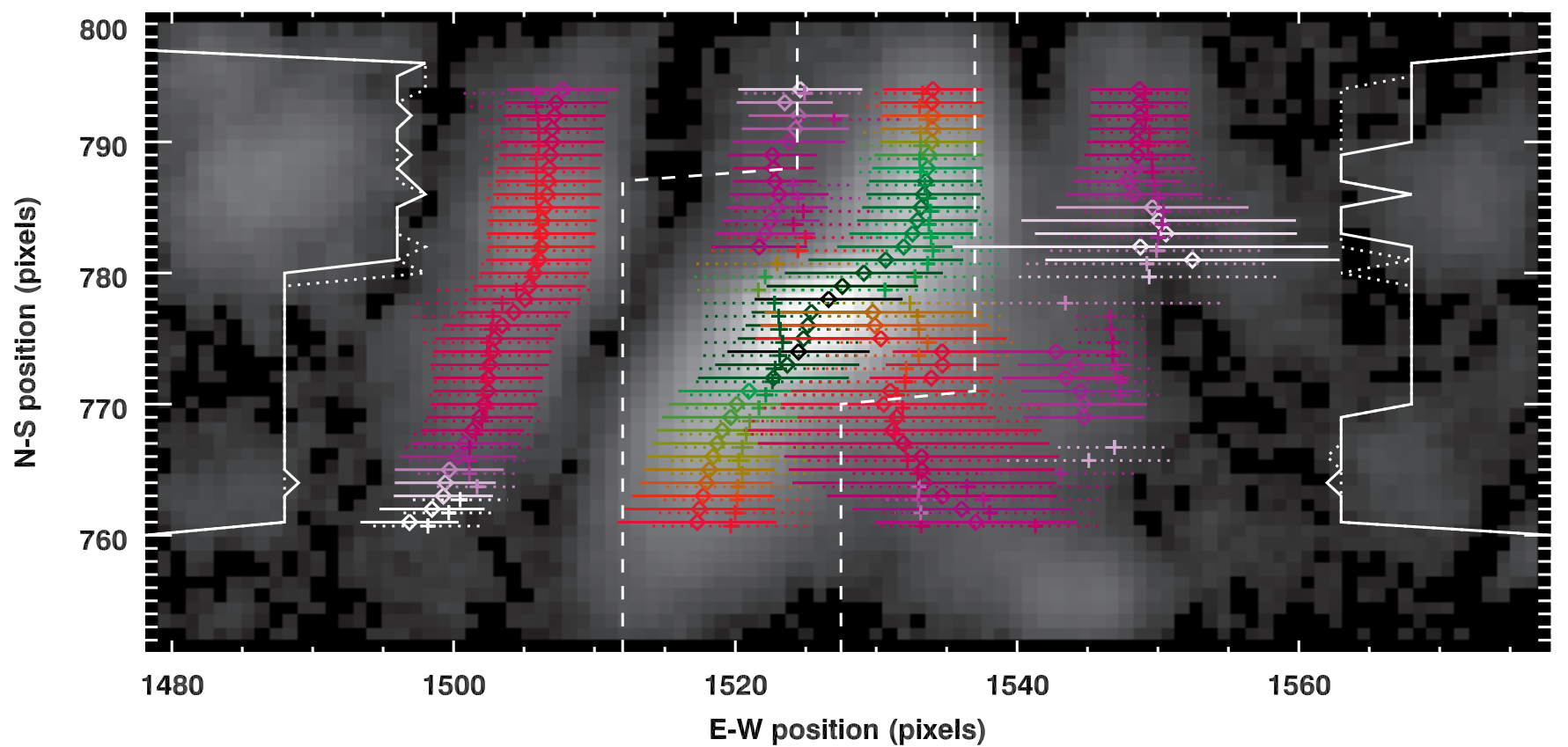

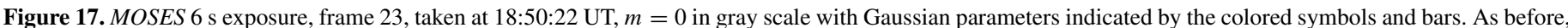

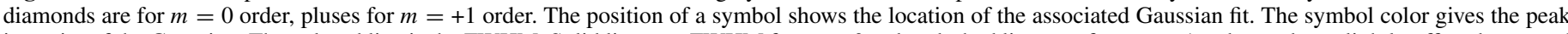

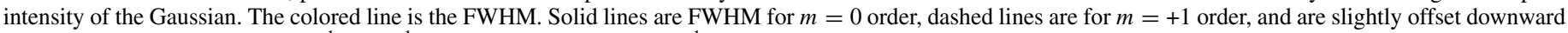
for clarity. Dispersion is $29 \mathrm{~km} \mathrm{~s}^{-1}$ pixel $^{-1}$. Plate scale is $440 \mathrm{~km} \mathrm{pixel}^{-1}$.

larger width than the $m=0$ Gaussian. Refer to Figure 10 . Likewise in [2,2] component fits, the $m=+1$ order fits would be wider than the $m=0$ order fits (and both by the same amount at that), but they would not be shifted in opposite directions. The error bars in Figure 18 are derived from the fit errors using textbook error propagation techniques. Like the errors in the fit parameters they are overestimates, particularly since the normal quadrature summation used in error propagation is only valid for statistically independent variables, and our fit parameters are likely anti-correlated. The large jumps in the size of errors seen in the figure indicate where the dashed bars (Figure 17) pick up (or drop) multiple Gaussians. Compositing multiple Gaussians greatly amplifies the fit errors.

Figure 19 shows the inferred Doppler broadening calculated by subtracting the $m=0$ composite width (from Equation (6)) from the $m=+1$ composite width in quadrature. Both widths are scaled by a factor of 2.3548 for comparison to FWHM line widths. As in Figure 18, the composite widths are calculated only for fits with centers falling between the dashed bars in Figure 17. The Doppler width in the core is non-thermal. Our instrumental line width has not been measured, however, the $\sim 50 \mathrm{~km} \mathrm{~s}^{-1}$ median width seen in the jets is an upper bound. The maximum Doppler width is $380 \mathrm{~km} \mathrm{~s}^{-1}$ FWHM at coordinates

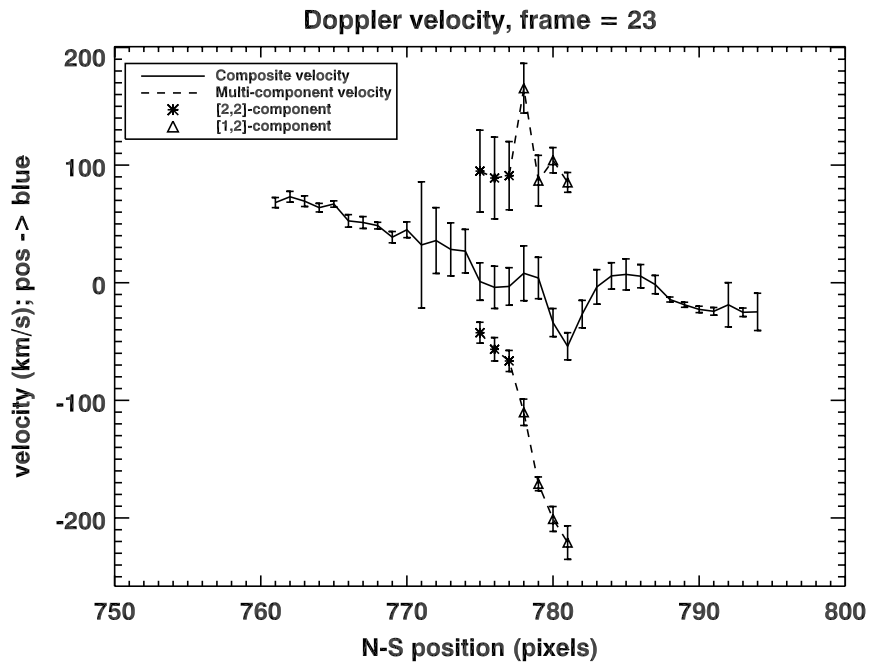

Figure 18. Doppler shift as a function of $y$-axis position. Composite centroids of the event core are calculated using Equation (5). Multi-component shifts are calculated in the explosive region where the $m=+1$ order broadening can be resolved into separate Gaussians. [1, 2]-component shifts show single-fit to double-fit shifts; that is, splitting of a single $m=0$ Gaussian into two oppositely directed Gaussians in $m=+1$. [2, 2]-component shifts have two-Gaussian fits in $m=0$, which spread apart when viewed in $m=+1$. 
Table 1

Fit Data for the Three Cases Shown in Figures 11-16

\begin{tabular}{|c|c|c|c|c|c|}
\hline Location & Order & Center (pixel) & Width (pixel) & Intensity $\left(\mathrm{DN} \mathrm{s}^{-1}\right)$ & Null Conf. $(\sigma)$ \\
\hline $\begin{array}{l}\text { Line } 768 \\
\text { (SE Jet) }\end{array}$ & $\begin{array}{l}m=0 \\
m=+1\end{array}$ & $\begin{aligned} 1501.34 & \pm 0.20 \\
1519.08 & \pm 0.05 \\
1531.17 & \pm 0.82 \\
1501.57 & \pm 0.34 \\
1520.77 & \pm 0.08 \\
1531.3 & \pm 1.5\end{aligned}$ & $\begin{array}{l}7.33 \pm 0.47 \\
9.28 \pm 0.21 \\
21.1 \pm 1.34 \\
8.80 \pm 0.80 \\
9.39 \pm 0.41 \\
20.4 \pm 2.2\end{array}$ & $\begin{aligned} 554 & \pm 46.0 \\
3860 & \pm 143 \\
2700 & \pm 187 \\
698 & \pm 81.2 \\
3810 & \pm 301 \\
2870 & \pm 354\end{aligned}$ & $\begin{array}{r}12.0 \\
27.0 \\
14.5 \\
8.6 \\
12.6 \\
8.1\end{array}$ \\
\hline $\begin{array}{l}\text { Line } 781 \\
\text { (Core) }\end{array}$ & $\begin{array}{l}m=0 \\
m=+1\end{array}$ & $\begin{aligned} 1506.07 & \pm 0.14 \\
1530.68 & \pm 0.07 \\
1552.5 & \pm 2.5 \\
1505.90 & \pm 0.16 \\
1522.97 & \pm 0.49 \\
1533.65 & \pm 0.29 \\
1549.2 & \pm 2.8\end{aligned}$ & $\begin{array}{l}7.48 \pm 0.33 \\
11.0 \pm 0.15 \\
20.9 \pm 7.2 \\
7.84 \pm 0.38 \\
11.5 \pm 0.79 \\
10.2 \pm 0.60 \\
17.1 \pm 6.0\end{array}$ & $\begin{aligned} 1480 & \pm 86.6 \\
7040 & \pm 120 \\
484 & \pm 196 \\
1600 & \pm 99 \\
4240 & \pm 340 \\
5080 & \pm 380 \\
607 & \pm 233\end{aligned}$ & $\begin{array}{r}17.1 \\
58.6 \\
2.5 \\
16.2 \\
12.6 \\
13.3 \\
2.6\end{array}$ \\
\hline $\begin{array}{l}\text { Line } 792 \\
\text { (NW Jet) }\end{array}$ & $\begin{array}{l}m=0 \\
m=+1\end{array}$ & $\begin{aligned} 1507.16 & \pm 0.18 \\
1524.48 & \pm 0.58 \\
1534.00 & \pm 0.08 \\
1548.72 & \pm 0.25 \\
1505.99 & \pm 0.16 \\
1527.0 & \pm 2.0 \\
1533.66 & \pm 0.17 \\
1549.12 & \pm 0.17\end{aligned}$ & $\begin{aligned} 7.23 & \pm 0.42 \\
7.09 & \pm 1.37 \\
7.29 & \pm 0.20 \\
7.08 & \pm 0.59 \\
8.27 & \pm 0.37 \\
9.9 & \pm 2.7 \\
6.74 & \pm 0.26 \\
6.68 & \pm 0.40\end{aligned}$ & $\begin{aligned} 635 & \pm 49.1 \\
281 & \pm 63.0 \\
2050 & \pm 65.1 \\
445 & \pm 49.0 \\
813 & \pm 47.7 \\
596 & \pm 197 \\
2120 & \pm 201 \\
545 & \pm 42.6\end{aligned}$ & $\begin{array}{r}12.9 \\
4.5 \\
31.5 \\
9.1 \\
17.1 \\
3.0 \\
10.5 \\
12.8\end{array}$ \\
\hline
\end{tabular}

Notes. Gaussian centers and widths are given in pixel units. Intensity in this context means the total integrated area under the Gaussian curve. The term "null confidence" refers to the zero intensity null hypothesis and is defined in the text.

$(1527,779)$ close to the location of peak intensity in the event. This width and the other widths in the core well in excess of $100 \mathrm{~km} \mathrm{~s}^{-1}$ are much too large to be thermally derived, given the He II thermal width of $30 \mathrm{~km} \mathrm{~s}^{-1}$ FWHM (Andretta et al. 2000). As in Figure 18, the error bars are calculated from propagation of the fit parameter errors. Some of the errors are (very obviously) spuriously large. These again occur at the places where the centroid limits (dashed bars in Figure 17) first pick up multiple Gaussians. These new Gaussians are far away, with weaker intensity, and large fit errors, contributing to a high error estimate. Points that appear without error bars are identically zero and show where we have fits in $m=0$ order which are slightly wider than the corresponding $m=+1$ order fits, giving an imaginary inferred Doppler width.

Between these three figures, we can identify the major components of the event as follows.

1. The core, approximately $4000 \mathrm{~km} \mathrm{E}-\mathrm{W}$ by $4800 \mathrm{~km} \mathrm{~N}-\mathrm{S}$, extends roughly across pixels $1523<x<1532$ and $772<y<783$. It is characterized by an increased Doppler broadening as shown in Figure 19 and a clearly resolved bi-directional Doppler shift shown in Figure 18. The locations of maximum blue- and redshift components are offset $1300 \mathrm{~km}$ in the $y$-direction and $1800 \mathrm{~km}$ in the $x$-direction.

2. The jets, each $\approx 6000 \mathrm{~km}$ in length, by contrast have little or no broadening and show only a one-sided Doppler shift. They are offset from each other in the E-W direction about $\approx 5700 \mathrm{~km}$.

3. The SE jet has a large blue Doppler shift, peaking at $70-80 \mathrm{~km} \mathrm{~s}^{-1}$, comparable to its sky-plane velocity (see Section 3.1). Taken together, these measurements imply a total velocity of $\approx 104 \mathrm{~km} \mathrm{~s}^{-1}$ at an angle to the plane of the sky of $46^{\circ}$.

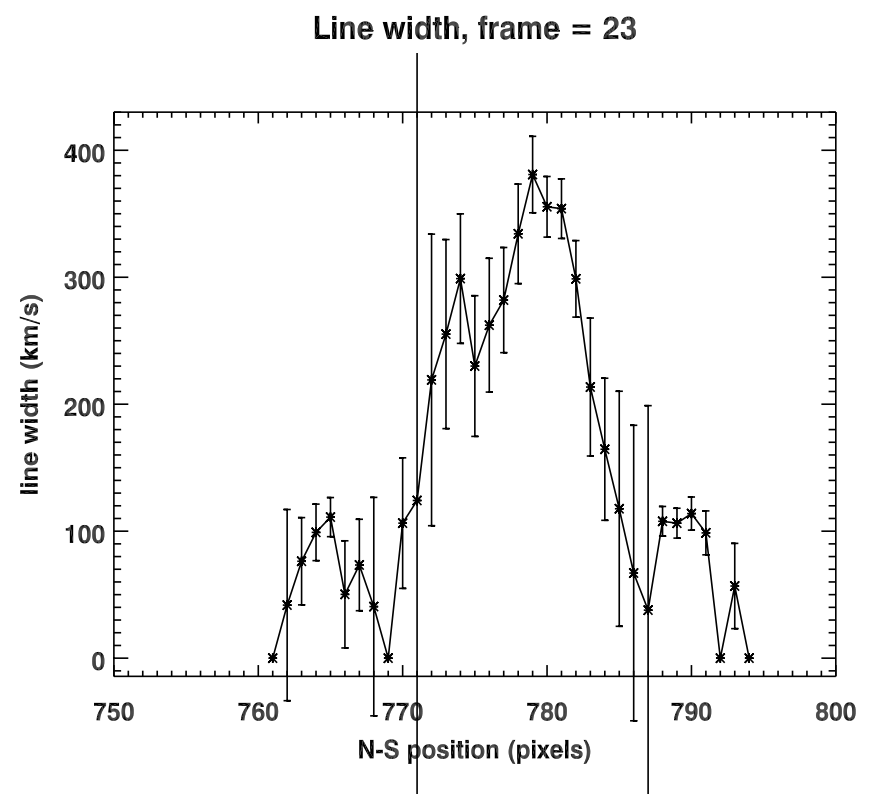

Figure 19. Inferred line width calculated from the quadrature difference of composite width from $m=0$ to $m=+1$ as a function of $y$-axis position. Composite widths are defined and calculated in a standard-deviation sense in Equation (6) but are scaled to FWHM in the figure and as quoted in the text.

4. The NW jet redshift is smaller, at about $20-30 \mathrm{~km} \mathrm{~s}^{-1}$. However, its sky-plane velocity is somewhat larger. The total velocity is $\approx 86 \mathrm{~km} \mathrm{~s}^{-1}$ at an angle with respect to the sky of $20^{\circ}$.

5. "Crossover points," locations where the sign of the jet Doppler shift appears to change, can be seen in Figure 17 at $(1523,772)$ and $(1533,786)$ where the jets merge with the core. 
6. The three-dimensional velocity vectors in the jets are

$$
\begin{aligned}
v_{\mathrm{NW}} & =(12 \pm 10.2,80 \pm 13.8,-25 \pm 5) \mathrm{km} \mathrm{s}^{-1} \\
v_{\mathrm{SE}} & =(17 \pm 10.3,-70 \pm 10.6,75 \pm 5) \mathrm{km} \mathrm{s}^{-1},
\end{aligned}
$$

giving an angle of $145^{\circ} .76 .9$ between the jets. The $+x$-direction is solar $\mathrm{W},+y$ is solar $\mathrm{N}$, and $+z$ is toward the observer. The velocity errors for the line-of-sight component are taken from Figure 18. Those for the components transverse to the line of sight are estimated from our visual estimate of the sky-plane jet positions, which was repeated three times using different image scaling. The quoted errors correspond to somewhat more than 0.5 pixel deviations in sky-plane position in each of the exposures used to compute the center-difference sky-plane velocity (see Section 3.1, Figures 8 and 9). The error in the angle between the jets is calculated from the velocity component errors via Monte Carlo simulation. The jet-angle histogram resulting from the simulation is nearly Gaussian, so the " \pm " notation has the usual meaning. We also use Monte Carlo simulation to calculate the confidence in excluding the anti-parallel jets null hypothesis. This is done by adjusting the jet velocities to force a $180^{\circ}$ angle while holding the magnitudes constant and, applying the same velocity errors as above, asking the question "what fraction of simulated velocity measurements yield an angle of $145^{\circ} .7$ or less?" The geometry of this null hypothesis can be constructed in a variety of ways, but in each of 13 cases tested the confidence is about $99.9 \%$.

\section{DISCUSSION AND CONCLUSIONS}

The He II event observed by MOSES-06 has all the features of a transition region $\mathrm{EE}$, and is within the upper range of EE parameters, such as size, lifetime, Doppler velocity, maximum broadening, etc., found in previous observations of EEs, as extensively cited in Section 1. It lies above a pair of converging, canceling magnetic poles. This suggests reconnection as a mechanism. The jets are expelled from the core with velocities of the order of transition region Alfvén velocities, a further suggestion of reconnection. The Doppler shifts derived in Section 4 show that the material is actually moving and rules out the hypothesis that the sky-plane velocities are only the apparent motion of plasma heating/cooling progressively outward through the He II band. EEs have been previously explained by a two-dimensional $X$-point reconnection model (see, for example, Innes et al. 1997, Figure 2(b)). That model predicts opposing collinear outflow jets. We see non-collinear jets, offset by $5700 \mathrm{~km}$, which corresponds to the entire width of the core. Furthermore, the jets are not anti-parallel, with an angle of $146^{\circ}$ between their flow directions.

Reconnection is a localized phenomenon. The velocities of reconnection jets need not be anti-parallel (although the illustrations are usually drawn that way), but they should have a common origin. We should be able to trace them back to an intersection where the reconnection which produced them occurred. We cannot do that here. We do not know what kind of reconnection process can produce jets which appear to be so far apart. One hypothesis is that the jets we see are not the jets of a single reconnection but are produced by separate reconnection events. We might interpret the noted crossover points as the source of each reconnection. In that case, the following questions occur: Where is the other half of each jet? Why are the velocities heading into the core (presumably a region of higher density and temperature) higher than the velocities heading away from the core? What processes associate the reconnections with each other? Does one reconnection "trigger" the other? The Alfvén crossing time of the core is $\approx 60 \mathrm{~s}$, yet the two jets appear simultaneously in our observations. Are both jets triggered by a preceding reconnection event in the core? What physical property provides the time coincidence of the jets? These considerations lead us to prefer the simpler idea of a single reconnection event, though a self-consistent interpretation has so far proved elusive.

It is also hard to see how a "near" potential field configuration could give rise to this kind of geometry (compare Longcope 1998). The presence of a filament nearby has been noted in Figure 8. We suspect this filament strongly influences the structure of the EE analyzed here. Filaments are associated with highly non-potential fields. One of the underlying magnetic poles (N47) is also coming from the region of the filament. Furthermore, N47 is a minority polarity as it is on the positive side of the filament.

Our observation of this event's three-dimensional structure and dynamics is only possible because of the simultaneous imaging and spectroscopy provided by MOSES. With an imager only, we would not know this is a transition region EE. With a slit spectrometer, we would have missed pieces of the full event, perhaps only seeing one of the jets, or only the core, depending on slit alignment, and would not have observed the peculiar, non-collinear geometry of the jets.

We gratefully acknowledge the assistance of Dana Longcope in obtaining and understanding the magnetic context data. This work was supported by NASA LCAS grants NAG5-10997 and NNX-07AG6G. J.L.F. was supported in part by NASA GSRP grant NGT5-50471, and by the Montana Space Grant Consortium fellowship program.

Facility: SOHO (MDI)

\section{APPENDIX}

\section{ALIGNMENT}

After dark subtraction, the images from the three spectral orders must be co-aligned as accurately as possible. All spectral measurements depend on this step. We use a low-order polynomial coordinate transformation for coregistration, allowing for the slight distortion that is present in the outboard $(m= \pm 1)$ images:

$$
\begin{aligned}
x^{\prime} & =\sum_{i=0}^{1} \sum_{j=0}^{1} a_{i j} x^{i} y^{j}, \\
y^{\prime} & =\sum_{i=0}^{1} \sum_{j=0}^{1} b_{i j} x^{i} y^{j} .
\end{aligned}
$$

The images were deemed aligned when the sum of the square of the residuals, normalized by intensity (in the manner of $\chi_{R}^{2}$ ), was minimized as a function of the polynomial warping coefficients $a_{i j}$ and $b_{i j} .{ }^{4}$ By aligning the three orders, we effectively assume a zero mean Doppler shift over the FOV; the measurement of non-zero mean Doppler shift would require calibration of the

\footnotetext{
4 In this application, the most consistent results are found when $\chi_{R}^{2}$ is normalized by the number of overlapping pixels, which is itself a function of the alignment parameters.
} 
instrument with an extremely well-collimated EUV line source, which was not available to us. The alignments of the three spectral orders were repeatable from exposure to exposure, to better than 0.2 pixel rms. No measurable relative movement of the detectors should occur in flight, so the median alignment across all of the $3 \mathrm{~s}$ exposures was taken as the alignment for the entire sequence.

\section{REFERENCES}

Andretta, V., Jordan, S. D., Brosius, J. W., Davila, J. M., Thomas, R. J., Behring,

W. E., Thompson, W. T., \& Garcia, A. 2000, ApJ, 535, 438

Arnaud, M., \& Rothenflug, R. 1985, A\&AS, 60, 425

Brosius, J. W., Davila, J. M., \& Thomas, R. J. 1998, ApJS, 119, 255

Brueckner, G. E., \& Bartoe, J.-D. F. 1983, ApJ, 272, 329

Brueckner, G. E., Bartoe, J.-D. F., Cook, J. W., Dere, K. P., Socker, D., Kurokawa, H., \& McCabe, M. 1988, ApJ, 335, 986

Bruner, M. E., Brown, W. A., \& Appert, K. L. 1989, Proc. SPIE, 1160, 620

Cook, J. W., \& Brueckner, G. E. 1991, in Solar Interior and Atmosphere, ed. A. N. Cox, W. C. Livingston, \& M. S. Matthews (Tucson, AZ: Univ. Arizona Press), 996

Culhane, J. L., et al. 2007, Sol. Phys., 243, 19

Dere, K. P. 1994, Adv. Space Res., 14, 13
Dere, K. P., Bartoe, J.-D. F., \& Brueckner, G. E. 1989, Sol. Phys., 123, 41 Dere, K. P., Bartoe, J.-D. F., Brueckner, G. E., Ewing, J., \& Lund, P. 1991, J. Geophys. Res., 96, 9399

Dere, K. P., Doschek, G. A., Mariska, J. T., Hansteen, V. H., Harra, L. K., Matsuzaki, K., \& Thomas, R. J. 2007, PASJ, 59, 721

Fox, J. L., \& Kankelborg, C. C. 2002, BAAS, 34, 733

Fox, J. L., Kankelborg, C. C., \& Metcalf, T. R. 2003, Proc. SPIE, 5157, 124

Freeland, S. L., \& Handy, B. N. 1998, Sol. Phys., 182, 497

Innes, D. E., Inhester, B., Axford, W. I., \& Willhelm, K. 1997, Nature, 386, 811

Kankelborg, C. C., \& Fox, J. L. 2004, BAAS, 36, 794

Kankelborg, C. C., Longcope, D. W., \& Martens, P. C. H. 2000, BAAS, 32, 829

Kankelborg, C. C., \& Thomas, R. J. 2001, Proc. SPIE, 4498, 16

Kankelborg, C. C., Walker, A. B. C., II., \& Hoover, R. B. 1997, ApJ, 491, 952

Kankelborg, C. C., Walker, A. B. C., II, Hoover, R. B., \& Barbee, T. W., Jr. 1996, ApJ, 466, 529

Kosugi, T., et al. 2007, Sol. Phys., 243, 3

Longcope, D. W. 1998, ApJ, 507, 433

Moses, D., et al. 1994, ApJ, 430, 913

Owens, S. M., et al. 2005, Proc. SPIE, 5900, 5

Press, W. H., Teukolsky, S. A., Vetterling, W. T., \& Flannery, B. P. 1992, Numerical Recipes in C. The Art of Scientific Computing (2nd ed; Cambridge: Cambridge Univ. Press)

Thomas, R. J., \& Kankelborg, C. C. 2001, BAAS, 33, 1434

Thomas, R. J., \& Neupert, W. M. 1994, ApJS, 91, 461

Vernazza, J. E., \& Reeves, E. M. 1978, ApJS, 37, 485 